ANNALES

POLONICI MATHEMATICI

$82.2(2003)$

\title{
Natural operators lifting linear vector fields from a vector bundle to its $r$-jet prolongations
}

\author{
by WŁOdZimierz M. Mikulski (Kraków)
}

\begin{abstract}
All natural operators $A$ transforming a linear vector field $X$ on a vector bundle $E$ into a vector field $A(X)$ on the $r$-jet prolongation $J^{r} E$ of $E$ are given. Similar results are deduced for the $r$-jet prolongations $J_{\mathrm{v}}^{r} E$ and $J^{[r]} E$ in place of $J^{r} E$.
\end{abstract}

0. Introduction. One of the problems studied in differential geometry is the naturality problem: how a geometrical object can induce canonically another geometrical object. An illustration is the Levi-Civita connection induced by a Riemannian tensor. Another example is the flow prolongation of a vector field to the tangent bundle. Such constructions appear in the context of natural operators in the sense of [3].

Natural operators $A: T \rightsquigarrow T F$ lifting vector fields $X$ on a manifold $M$ to a natural bundle $F M$ are used practically in all papers in which the problem of prolongations of geometric structures is studied. That is why such natural operators are classified in [1]-[5] and other papers.

Natural operators $A: T_{\text {proj }} \rightsquigarrow T F$ lifting projectable vector fields $X$ on a fibered (or fibered-fibered) manifold $Y$ to a natural bundle $F Y$ are important for the same reasons as above. The most important $F Y$ is the $r$-jet prolongation bundle $J^{r} Y$ (or the $(r, s, q)$-prolongation bundle $J^{(r, s, q)} Y$ ). It plays a fundamental role in the theory of connections, differential equations and Lagrangians.

The authors of [3] studied the naturality problem how a projectable vector field $X$ on a fibered manifold $p: Y \rightarrow M$ can induce a vector field $A(X)$ on the $r$-jet prolongation $J^{r} Y$ of $Y$. This problem was reflected in the concept of $\mathcal{F} \mathcal{M}_{m, n}$-natural operators $A: T_{\text {proj } \mid \mathcal{F} \mathcal{M}_{m, n}} \rightsquigarrow T J^{r}$. It was proved that any $\mathcal{F} \mathcal{M}_{m, n}$-natural operator $A: T_{\operatorname{proj} \mid \mathcal{F} \mathcal{M}_{m, n}} \rightsquigarrow T J^{r}$ is a constant multiple of the flow operator $\mathcal{J}^{r}: T_{\text {proj| } \mathcal{F} \mathcal{M}_{m, n}} \rightsquigarrow T J^{r}$.

2000 Mathematics Subject Classification: 58A05, 58A20.

Key words and phrases: vector gauge bundle functors, natural operators, jets. 
In [5], we extended this result to the $(r, s, q)$-jet prolongation functor $J^{(r, s, q)}$ on fibered-fibered manifolds and obtained a similar classification of natural operators lifting projectable-projectable vector fields to $J^{(r, s, q)}$.

The results of [3] and [5] show that the bundles $J^{r} Y$ and $J^{(r, s, q)} Y$ are "poor" with respect to liftings of projectable vector fields. This indicates that there is not much possibility to prolong geometric objects by means of liftings of projectable vector fields.

It seems interesting to solve problems similar to those in [3] and [5] for the $r$-jet prolongation bundle $J^{r} E$ of a vector bundle $E$ in place of a fibered manifold $Y$. The bundle $J^{r} E$ plays a similar role to $J^{r} Y$. It is fundamental in the theory of linear connections, linear differential equations and linear Lagrangians. Therefore the possibility of lifting geometric objects to $J^{r} E$ by means of lifts of linear vector fields is interesting. We prove that this possibility is rather limited.

In the first part of the present paper we study the naturality problem of how a linear vector field $X$ on a vector bundle $E$ can induce a vector field $A(X)$ on the $r$-jet prolongation $J^{r} E$ of $E$. This problem is reflected in the concept of $\mathcal{V} \mathcal{B}_{m, n}$-natural operators $A: T_{\text {lin } \mid \mathcal{V} \mathcal{B}_{m, n}} \rightsquigarrow T J^{r}$ lifting a linear vector field $X$ on $E$ to a vector field $A(X)$ on $J^{r} E$. We prove that for integers $m \geq 2, n \geq 1$ and $r \geq 0$ any such operator is a linear combination of the flow operator $\mathcal{J}^{r} X$ and the Liouville vector field $L$ on $J^{r} E$. As corollaries, we derive similar results for $J^{r} E^{*},\left(J^{r} E\right)^{*}$ and $\left(J^{r} E^{*}\right)^{*}$ in place of $J^{r} E$.

In the second part of the paper we study the similar problem for the vertical $r$-jet prolongation $J_{\mathrm{v}}^{r} E$ in place of $J^{r} E$. We prove that for integers $m \geq 2, n \geq 1$ and $r \geq 1$ any $\mathcal{V B}_{m, n}$-natural operator lifting a linear vector field $X$ on $E$ to a vector field on $J_{\mathrm{v}}^{r} E$ is a linear combination of the flow operator $\mathcal{J}^{r} X$, the Liouville vector field $L$ on $J_{\mathrm{v}}^{r} E$ and some explicitly constructed natural operators $V^{\langle s\rangle}(X)$ for $s=0, \ldots, r$. As corollaries, we derive similar results for $J_{\mathrm{v}}^{r} E^{*},\left(J_{\mathrm{v}}^{r} E\right)^{*}$ and $\left(J_{\mathrm{v}}^{r} E^{*}\right)^{*}$ in place of $J_{\mathrm{v}}^{r} E$.

In the third part we prove that for integers $m \geq 2, n \geq 1$ and $r \geq 1$, any $\mathcal{V} \mathcal{B}_{m, n}$-natural linear operator $A$ lifting a linear vector field $X$ from a vector bundle $E$ with $m$-dimensional base and $n$-dimensional fibers to a vector field $A(X)$ on the $[r]$-jet prolongation $J^{[r]} E$ of $E$ is a linear combination of the flow operator $\mathcal{J}^{[r]}$ and some explicitly constructed natural linear operator $U(X)$. As corollaries we deduce similar results for $J^{[r]} E^{*},\left(J^{[r]} E\right)^{*}$ and $\left(J^{[r]} E^{*}\right)^{*}$ in place of $J^{[r]} E$.

The category of vector bundles with $m$-dimensional bases and vector bundle maps with local diffeomorphisms as base maps will be denoted by $\mathcal{V} \mathcal{B}_{m}$.

The category of vector bundles with $m$-dimensional bases and $n$-dimensional fibers and vector bundle isomorphisms onto open vector subbundles will be denoted by $\mathcal{V} \mathcal{B}_{m, n}$. 
The trivial vector bundle $\mathbb{R}^{m} \times \mathbb{R}^{n}$ over $\mathbb{R}^{m}$ with standard fiber $\mathbb{R}^{n}$ will be denoted by $\mathbb{R}^{m, n}$.

The coordinates on $\mathbb{R}^{m}$ will be denoted by $x^{1}, \ldots, x^{m}$, and the fiber coordinates on $\mathbb{R}^{m, n}$ by $y^{1}, \ldots, y^{n}$.

All manifolds are assumed to be finite-dimensional and smooth. Maps are assumed to be smooth, i.e. of class $\mathcal{C}^{\infty}$.

\section{NATURAL OPERATORS LIFTING LINEAR VECTOR FIELDS FROM A VECTOR BUNDLE TO ITS $r$-JET PROLONGATION}

1. The $r$-jet prolongation functor. Given a $\mathcal{V} \mathcal{B}_{m}$-object $p: E \rightarrow M$ the $r$-jet prolongation $J^{r} E$ of $E$ is the vector bundle

$$
J^{r} E=\left\{j_{x}^{r} \sigma \mid \sigma \text { is a local section of } E, x \in M\right\}
$$

over $M$. Every $\mathcal{V} \mathcal{B}_{m}$-map $f: E_{1} \rightarrow E_{2}$ covering $f: M_{1} \rightarrow M_{2}$ induces a vector bundle map $J^{r} f: J^{r} E_{1} \rightarrow J^{r} E_{2}$ covering $\underline{f}$ such that

$$
J^{r} f\left(j_{x}^{r} \sigma\right)=j_{\underline{f}(x)}^{r}\left(f \circ \sigma \circ \underline{f}^{-1}\right), \quad j_{x}^{r} \sigma \in J^{r} E_{1} .
$$

The functor $J^{r}: \mathcal{V B}_{m} \rightarrow \mathcal{V} \mathcal{B}_{m}$ is a fiber product preserving vector gauge bundle functor.

From now on we identify $J_{0}^{r}\left(\mathbb{R}^{m, n}\right)$ and $\times^{n} J_{0}^{r}\left(\mathbb{R}^{m}, \mathbb{R}\right)$ via $j_{0}^{r}(x, \sigma(x)) \mapsto$ $\left(j_{0}^{r} \sigma^{l}\right)_{l=1}^{n}, \sigma=\left(\sigma^{1}, \ldots, \sigma^{n}\right): \mathbb{R}^{m} \rightarrow \mathbb{R}^{n}$.

2. Examples of natural operators $T_{\text {lin } \mid \mathcal{V} \mathcal{B}_{m, n}} \rightsquigarrow T J^{r}$. Let $p: E \rightarrow M$ be a $\mathcal{V} \mathcal{B}_{m, n}$-object. A projectable vector field $X$ on $E$ is called linear if $X: E \rightarrow T E$ is a vector bundle map from $p: E \rightarrow M$ into $T p: T E \rightarrow T M$. Equivalently, the flow $\mathrm{Fl}_{t}^{X}$ of $X$ is formed by $\mathcal{V} \mathcal{B}_{m, n}$-maps. The space of linear vector fields on $E$ will be denoted by $\mathcal{X}_{\text {lin }}(E)$.

A natural operator $A: T_{\operatorname{lin} \mid \mathcal{V B}} \mathcal{B}_{m, n} \rightsquigarrow T J^{r}$ is a $\mathcal{V} \mathcal{B}_{m, n}$-invariant family of regular operators $A: \mathcal{X}_{\text {lin }}(E) \rightarrow \mathcal{X}\left(J^{r} E\right)$ for any $\mathcal{V} \mathcal{B}_{m, n}$-object $E$. The $\mathcal{V} \mathcal{B}_{m, n}$-invariance means that for any $\mathcal{V B}_{m, n}$-map $f: E_{1} \rightarrow E_{2}$ and any $f$-conjugate linear vector fields $X$ and $Y$ on $E_{1}$ and $E_{2}$ the vector fields $A(X)$ and $A(Y)$ are $J^{r} f$-conjugate. The regularity means that $A$ transforms smoothly parametrized families of linear vector fields into smoothly parametrized families of vector fields.

EXAMPLE 1 (The flow operator). Let $X$ be a linear vector field on a $\mathcal{V} \mathcal{B}_{m, n}$-object $p: E \rightarrow M$. The flow $\mathrm{Fl}_{t}^{X}$ of $X$ is formed by $\mathcal{V} \mathcal{B}_{m, n}$-maps on $E$. Applying the functor $J^{r}$ we obtain a flow $J^{r}\left(\mathrm{Fl}_{t}^{X}\right)$ on $J^{r} E$. The vector field $\mathcal{J}^{r} X$ on $J^{r} E$ corresponding to $J^{r}\left(\mathrm{Fl}_{t}^{X}\right)$ is called the flow prolongation of $X$. The correspondence $\mathcal{J}^{r}: T_{\text {lin } \mid \mathcal{V} \mathcal{B}_{m, n}} \rightsquigarrow T J^{r}, X \mapsto \mathcal{J}^{r} X$, is a natural operator. 
EXAmple 2 (The Liouville vector field). Let $p: E \rightarrow M$ be a $\mathcal{V} \mathcal{B}_{m, n^{-}}$ object. Let $L$ be the Liouville vector field on the vector bundle $J^{r} E, L_{y}=$ $y \in J_{x}^{r} E \cong T_{y}\left(J_{x}^{r} E\right) \subset T_{y} J^{r} E, y \in J_{x}^{r} E, x \in M$. The correspondence $L: T_{\operatorname{lin} \mid \mathcal{V} \mathcal{B}_{m, n}} \rightsquigarrow T J^{r}, X \mapsto L$, is a natural operator.

3. The main result of the first part. The main result in the first part is the following classification theorem.

Theorem 1. Let $m \geq 2, n \geq 1$ and $r \geq 0$ be integers. Any natural operator $A: T_{\operatorname{lin} \mid \mathcal{V} \mathcal{B}_{m, n}} \rightsquigarrow T J^{r}$ is a linear combination with real coefficients of the flow operator $\mathcal{J}^{r}$ and the Liouville vector field $L$.

The proof will occupy Sections 4-10.

Corollary 1. Let $m \geq 2, n \geq 1$ and $r \geq 0$ be integers. Any natural linear operator $A: T_{\operatorname{lin} \mid \mathcal{V} \mathcal{B}_{m, n}} \rightsquigarrow T J^{r}$ is a constant multiple of the flow operator.

\section{A reducibility lemma}

Lemma 1. Every natural operator $A: T_{\operatorname{lin} \mid \mathcal{V} \mathcal{B}_{m, n}} \rightsquigarrow T J^{r}$ is uniquely determined by the restriction $\widetilde{A}=A\left(\frac{\partial}{\partial x^{1}}\right) \mid J_{0}^{r}\left(\mathbb{R}^{m, n}\right)$ of $A\left(\frac{\partial}{\partial x^{1}}\right)$ to the fiber $J_{0}^{r}\left(\mathbb{R}^{m, n}\right)$ of $J^{r}\left(\mathbb{R}^{m, n}\right)$ over $0 \in \mathbb{R}^{m}$.

Proof. The lemma follows in a standard way from the regularity and invariance of $A$ with respect to $\mathcal{V} \mathcal{B}_{m, n}$-morphisms and the fact that any linear vector field $X$ on $p: E \rightarrow M$ covering a non-vanishing vector field on $M$ is locally $\mathcal{V} \mathcal{B}_{m, n}$-conjugate to $\frac{\partial}{\partial x^{1}}$.

\section{A decomposition lemma}

Lemma 2. Let $A: T_{\operatorname{lin} \mid \mathcal{V} \mathcal{B}_{m, n}} \rightsquigarrow T J^{r}$ be a natural operator. Then there exists $\alpha \in \mathbb{R}$ such that $A-\alpha \mathcal{J}^{r}$ is a vertical type operator.

Proof. Put $\widetilde{\widetilde{A}}=T \pi \circ \widetilde{A}: J_{0}^{r}\left(\mathbb{R}^{m, n}\right) \rightarrow T_{0} \mathbb{R}^{m}$, where $\widetilde{A}$ is as in Lemma 1 and $\pi: J^{r}\left(\mathbb{R}^{m, n}\right) \rightarrow \mathbb{R}^{m}$ is the bundle projection.

Using the invariance of $A$ with respect to the fiber homotheties $b_{\tau}$ for $\tau \neq 0$ and then letting $\tau \rightarrow 0$ we see that $\widetilde{\widetilde{A}}(y)=\widetilde{\widetilde{A}}(0)$ for any $y \in J_{0}^{r}\left(\mathbb{R}^{m, n}\right)$.

Write $\widetilde{\widetilde{A}}(0)=\sum_{i} \alpha_{i} \frac{\partial}{\partial x^{i} 0}$ for some $\alpha_{i} \in \mathbb{R}, i=1, \ldots, m$. Using the invariance of $A$ with respect to $a_{\tau}=\left(x^{1}, \tau x^{2}, \ldots, \tau x^{m}, y^{1}, \ldots, y^{n}\right)$ for $\tau \neq 0$ we deduce that $\alpha_{2}=\ldots=\alpha_{m}=0$. Then $\widetilde{\widetilde{A}}(y)=\alpha \frac{\partial}{\partial x^{1} 0}$ for any $y \in$ $J_{0}^{r}\left(\mathbb{R}^{m, n}\right)$, where $\alpha=\alpha_{1}$. Thus $\left(A-\alpha \mathcal{J}^{r}\right)\left(\frac{\partial}{\partial x^{1}}\right) \mid J_{0}^{r}\left(\mathbb{R}^{m, n}\right)$ is vertical. Hence $A-\alpha \mathcal{J}^{r}$ is vertical by Lemma 1 .

6. Natural operators $T_{\operatorname{lin} \mid \mathcal{V} \mathcal{B}_{m, n}} \rightsquigarrow T J^{r}$ of vertical type. By Lemma 2 , Theorem 1 is a consequence of the following fact. 
Proposition 1. Let $m \geq 2, n \geq 1$ and $r \geq 0$ be integers. Let $A$ : $T_{\operatorname{lin} \mid \mathcal{V} \mathcal{B}_{m, n}} \rightsquigarrow T J^{r}$ be a natural operator of vertical type. There exists $\gamma \in \mathbb{R}$ such that $A=\gamma L$.

The proof will occupy Sections 7-10.

\section{Some preparation}

Lemma 3. Let $A: T_{\text {lin } \mid \mathcal{V} \mathcal{B}_{m, n}} \rightsquigarrow T J^{r}$ be a natural operator of vertical type. Define $\bar{A}: J_{0}^{r}\left(\mathbb{R}^{m, n}\right) \rightarrow J_{0}^{r}\left(\mathbb{R}^{m, n}\right)$ by

$$
\widetilde{A}(y)=(y, \bar{A}(y)) \in J_{0}^{r}\left(\mathbb{R}^{m, n}\right) \times J_{0}^{r}\left(\mathbb{R}^{m, n}\right) \cong\left(V J^{r}\right)_{0}\left(\mathbb{R}^{m, n}\right),
$$

$y \in J_{0}^{r}\left(\mathbb{R}^{m, n}\right)$, where $\widetilde{A}$ is as in Lemma 1 . Then $A$ is uniquely determined by $\bar{A}$. Moreover, $\bar{A}$ is linear and it satisfies the following conditions:

(i) For any locally $\mathcal{V} \mathcal{B}_{m, n}-m a p f: \mathbb{R}^{m, n} \rightarrow \mathbb{R}^{m, n}$ preserving germ ${ }_{0}\left(\frac{\partial}{\partial x^{1}}\right)$,

$$
J_{0}^{r} f \circ \bar{A}=\bar{A} \circ J_{0}^{r} f .
$$

(ii) For any $\beta \in(\mathbb{N} \cup\{0\})^{m}$ with $|\beta| \leq r$ and $l=1, \ldots, n$,

$$
\bar{A}\left(0, \ldots, 0, j_{0}^{r} x^{\beta}, 0, \ldots, 0\right)=\left(\sum_{|\sigma| \leq|\beta|} c_{\sigma}^{\beta, l, k} j_{0}^{r} x^{\sigma}\right)_{k=1}^{n}
$$

for some $c_{\sigma}^{\beta, l, k} \in \mathbb{R}$, where the sums are over all $\sigma \in(\mathbb{N} \cup\{0\})^{m}$ with $|\sigma| \leq|\beta|,\left(0, \ldots, 0, j_{0}^{r} x^{\beta}, 0, \ldots, 0\right) \in J_{0}^{r}\left(\mathbb{R}^{m, n}\right), j_{0}^{r} x^{\beta}$ in position $l$.

Proof. Since $\widetilde{A}$ is uniquely determined by $\bar{A}, A$ is uniquely determined by $\bar{A}$ in view of of Lemma 1 .

By the invariance of $A$ with respect to the fiber homotheties we get the homogeneity condition $\bar{A}(\tau y)=\tau \bar{A}(y)$ for any $y \in J_{0}^{r}\left(\mathbb{R}^{m, n}\right)$ and $\tau \neq 0$. So, $\bar{A}$ is linear by the homogeneous function theorem.

From the invariance of $A$ with respect to a $\mathcal{V} \mathcal{B}_{m, n}$-map $f: \mathbb{R}^{m, n} \rightarrow \mathbb{R}^{m, n}$ preserving $\operatorname{germ}_{0}\left(\frac{\partial}{\partial x^{1}}\right)$ we obtain $J_{0}^{r} f \circ \bar{A}=\bar{A} \circ J_{0}^{r} f$.

For any $t \in \mathbb{R}$ define $\bar{A}_{t}: J_{0}^{r}\left(\mathbb{R}^{m, n}\right) \rightarrow J_{0}^{r}\left(\mathbb{R}^{m, n}\right)$ by $A\left(t \frac{\partial}{\partial x^{1}}\right)(y)=$ $\left(y, \bar{A}_{t}(y)\right), y \in J_{0}^{r}\left(\mathbb{R}^{m, n}\right)$. Clearly, $\bar{A}=\bar{A}_{1}$. Let $\beta \in(\mathbb{N} \cup\{0\})^{m}$ with $|\beta| \leq r$ and $l=1, \ldots, n$. We can write

$$
\bar{A}_{t}\left(0, \ldots, 0, j_{0}^{r} x^{\beta}, 0, \ldots, 0\right)=\left(\sum_{|\sigma| \leq r} c_{\sigma}^{\beta, l, k}(t) j_{0}^{r} x^{\sigma}\right)_{k=1}^{n}
$$

for some smooth maps $c_{\sigma}^{\beta, l, k}: \mathbb{R} \rightarrow \mathbb{R}$, where the sums are as in the statement and $j_{0}^{r} x^{\beta}$ is in position $l$. By the invariance of $A$ with respect to the base homotheties $\left(\tau x^{1}, \ldots, \tau x^{m}, y^{1}, \ldots, y^{n}\right)$ we obtain the homogeneity condition $c_{\sigma}^{\beta, l, k}(\tau t) \frac{1}{\tau^{|\beta|}}=c_{\sigma}^{\beta, l, k}(t) \frac{1}{\tau^{|\sigma|}}$ for $\tau \neq 0$. Thus $c_{\sigma}^{\beta, l, k}=0$ if $|\sigma|>|\beta|$. 
8. The main lemma. By Lemma $3, A$ is uniquely determined by $\bar{A}$. So, Proposition 1 is a consequence of the following lemma.

LeMmA 4. Let $m \geq 2, n \geq 1$ and $r \geq 0$ be integers. Let $\bar{A}: J_{0}^{r}\left(\mathbb{R}^{m, n}\right) \rightarrow$ $J_{0}^{r}\left(\mathbb{R}^{m, n}\right)$ be a linear map satisfying conditions (i) and (ii) of Lemma 3 . Then there is $\gamma \in \mathbb{R}$ such that $\bar{A}=\gamma \operatorname{id}_{J_{0}^{r}\left(\mathbb{R}^{m, n}\right)}$.

We will prove Lemma 4 by induction on $r$.

9. The case $r=0$. Let $r=0$. By the invariance of $\bar{A}$ with respect to changes of fibered coordinates (see condition (i)) we see that $\bar{A}$ is uniquely determined by $\bar{A}\left(j_{0}^{0} 1,0, \ldots, 0\right)$.

We can write $\bar{A}\left(j_{0}^{0} 1,0, \ldots, 0\right)=\left(c_{1} j_{0}^{0} 1, \ldots, c_{n} j_{0}^{0} 1\right)$. By the invariance of $\bar{A}$ with respect to $f=\left(x^{1}, \ldots, x^{m}, y^{1}, \tau y^{2}, \ldots, \tau y^{n}\right)$ (see condition (i)) we deduce that $c_{2}=\ldots=c_{n}=0$. Then $\bar{A}=\gamma \operatorname{id}_{J_{0}^{0}\left(\mathbb{R}^{m, n}\right)}$ with $\gamma=c_{1}$.

10. The inductive step. Assume that Lemma 4 is true for $r=r_{0}$. Let $r=r_{0}+1$.

SteP 1: A condition. Let

$$
\bar{A}\left(j_{0}^{r}\left(\left(x^{1}\right)^{r}\right), 0, \ldots, 0\right)=\left(\sum_{|\sigma| \leq r} c_{\sigma}^{k} j_{0}^{r} x^{\sigma}\right)_{k=1}^{n}
$$

for some $c_{\sigma}^{k} \in \mathbb{R}$. We prove that

$$
c_{(0, \ldots, 0)}^{1}=0 .
$$

Indeed, by the invariance of $\bar{A}$ with respect to the (locally defined) map $f=\left(x^{1}, \ldots, x^{m}, y^{1}+x^{2} y^{1}, y^{2}, \ldots, y^{n}\right)$ (see (i)) and (1) we obtain $c_{(0,1,0, \ldots, 0)}^{1}=c_{(0,1,0, \ldots, 0)}^{1}+c_{(0, \ldots, 0)}^{1}$, i.e. $(2)$.

STEP 2: A preparation. Let $\alpha \in(\mathbb{N} \cup\{0\})^{m},|\alpha|=r, l=1, \ldots, n$. We prove that

$$
B\left(0, \ldots, 0, j_{0}^{r} x^{\alpha}, 0, \ldots, 0\right)=\left(0, \ldots, 0, c j_{0}^{r} x^{\alpha}, 0, \ldots, 0\right)
$$

for some real $c$ (independent of $\alpha$ and $l$ ), where $j_{0}^{r} x^{\alpha}$ is in position $l$.

By the invariance of $\bar{A}$ with respect to (locally defined) $\left(x^{1}, \ldots, x^{i-1}, x^{i}+\right.$ $\left.\tau\left(x^{2}\right)^{2}, x^{i+1}, \ldots, x^{m}, y^{1}, \ldots, y^{n}\right)^{-1}$ for $\tau \in \mathbb{R}$ and $i=1, \ldots, m$ (see (i)), from (1) it follows that $\bar{A}\left(j_{0}^{r}\left(\left(x^{1}\right)^{r}\right), 0, \ldots, 0\right)=\left(\sum_{\sigma}\left(c_{\sigma}^{k} j_{0}^{r} x^{\sigma}+\tau \sigma_{i} c_{\sigma}^{k} j_{0}^{r} x^{\sigma-e_{i}+e_{2}+e_{2}}\right.\right.$ $+\ldots))_{k=1}^{n}$, where the dots stand for a finite sum of monomials in $\tau$ of degree $\geq 2$. (Observe that we have used $m \geq 2$.) Thus

$$
c_{\sigma}^{k}=0 \quad \text { for } 0<|\sigma|<r .
$$

Moreover, by the invariance of $\bar{A}$ with respect to $\left(x^{1}, \ldots, x^{m}, y^{1}, \tau y^{2}, \ldots, \tau y^{n}\right)$ (see (i)), for $\tau \neq 0$ we deduce that

$$
c_{\sigma}^{k}=0 \quad \text { for } k \neq 1 .
$$


Then by (2), (4) and (5) and the invariance of $\bar{A}$ with respect to $\left(x^{1}, \tau x^{2}, \ldots\right.$ $\left.\ldots, \tau x^{m}, y^{1}, \ldots, y^{n}\right)$ (see (i)) we deduce that

$$
\bar{A}\left(j_{0}^{r}\left(\left(x^{1}\right)^{r}\right), 0, \ldots, 0\right)=\left(c j_{0}^{r}\left(\left(x^{1}\right)^{r}\right), 0, \ldots, 0\right)
$$

for $c=c_{(r, 0, \ldots, 0)}^{1} \in \mathbb{R}$. Then using the invariance of $\bar{A}$ with respect to $\left(x^{1}+\right.$ $\left.\tau^{2} x^{2}+\ldots+\tau^{m} x^{m}, x^{2}, \ldots, x^{m}, y^{1}, \ldots, y^{n}\right)^{-1}$ for $\tau^{2}, \ldots, \tau^{m} \in \mathbb{R}$ (see (i)) we get

$$
\begin{aligned}
\bar{A}\left(j_{0}^{r}\left(\left(x^{1}+\tau^{2} x^{2}+\ldots+\tau^{m} x^{m}\right)^{r}\right), 0 \ldots, 0\right) \\
\quad=\left(c j_{0}^{r}\left(\left(x^{1}+\tau^{2} x^{2}+\ldots+\tau^{m} x^{m}\right)^{r}\right), 0, \ldots, 0\right) .
\end{aligned}
$$

Both sides of (7) are polynomials in $\tau$. Considering the coefficients of $\left(\tau^{2}\right)^{\alpha_{2}} \ldots\left(\tau^{m}\right)^{\alpha_{m}}$ we get

$$
\bar{A}\left(j_{0}^{r} x^{\alpha}, 0 \ldots, 0\right)=\left(c j_{0}^{r} x^{\alpha}, 0, \ldots, 0\right) .
$$

Then using the invariance of $\bar{A}$ with respect to permutations of fibered coordinates (see (i)) we get (3).

SteP 3: Using the inductive assumption. By Step 2 we have a linear map $[\bar{A}]: J_{0}^{r-1}\left(\mathbb{R}^{m, n}\right) \rightarrow J_{0}^{r-1}\left(\mathbb{R}^{m, n}\right)$ factorizing $\bar{A}$. By the assumptions (i) and (ii) on $\bar{A}$ we see that $[\bar{A}]$ satisfies (i) and (ii) for $r-1$. So, by the inductive assumption,

$$
[\bar{A}]=\gamma \operatorname{id}_{J_{0}^{r-1}\left(\mathbb{R}^{m, n}\right)}
$$

for some $\gamma \in \mathbb{R}$.

STEP 4: What remains to be proved. It remains to prove that $\bar{A}=$ $\gamma \operatorname{id}_{J_{0}^{r}\left(\mathbb{R}^{m, n}\right)}$, where $\gamma$ is as in (9).

By assumption (ii) on $\bar{A}$ and by equality (9) we have

$$
\bar{A}\left(0, \ldots, 0, j_{0}^{r} x^{\beta}, 0 \ldots, 0\right)=\gamma\left(0, \ldots, 0, j_{0}^{r} x^{\beta}, 0, \ldots, 0\right)
$$

for any $\beta \in(\mathbb{N} \cup\{0\})^{m}$ with $|\beta|<r$ and $l=1, \ldots, n$, where $j_{0}^{r} x^{\beta}$ is in position $l$. So, it remains to prove that for any $\alpha \in(\mathbb{N} \cup\{0\})^{m}$ with $|\alpha|=r$ and $l=1, \ldots, n$ we have

$$
\bar{A}\left(0, \ldots, 0, j_{0}^{r} x^{\alpha}, 0 \ldots, 0\right)=\gamma\left(0, \ldots, 0, j_{0}^{r} x^{\alpha}, 0, \ldots, 0\right) .
$$

Therefore, by Step 2 it remains to prove that $c=\gamma$, where $c$ is as in (3).

STEP 5: End of the proof. Using (10) for $\beta=(r-1,0, \ldots, 0) \in(\mathbb{N} \cup\{0\})^{m}$ and the invariance of $\bar{A}$ with respect to $\left(x^{1}+\left(x^{2}\right)^{2}, x^{2}, \ldots, x^{m}, y^{1}, \ldots, y^{n}\right)^{-1}$ (see (i)) we deduce that

$$
\bar{A}\left(j_{0}^{r}\left(\left(x^{1}\right)^{r-2}\left(x^{2}\right)^{2}\right), 0, \ldots, 0\right)=\gamma\left(j_{0}^{r}\left(\left(x^{1}\right)^{r-2}\left(x^{2}\right)^{2}\right), 0, \ldots, 0\right) .
$$

Now from (11) and (3) with $\beta=(r-2,2,0, \ldots, 0)$ we get $c=\gamma$.

11. Some versions of Theorem 1. By Theorem 1 , for any $\mathcal{V} \mathcal{B}_{m, n^{-}}$ natural operator $A$ lifting a linear vector field $X$ on a vector bundle $E$ to a 
vector field $A(X)$ on $J^{r} E$, the vector field $A(X)$ is always linear. We now prove this independently together with another similar fact.

Proposition 2. Let $A$ be a $\mathcal{V B}_{m, n}$-natural operator lifting a linear vector field $X$ on a vector bundle $E$ to a vector field $A(X)$ on $J^{r} E$ (or $J^{r} E^{*}$ or $\left(J^{r} E\right)^{*}$ or $\left.\left(J^{r} E^{*}\right)^{*}\right)$. Then $A(X)$ is always linear.

Proof. It is easy to see this for $X=\frac{\partial}{\partial x^{1}}$. (More precisely, the flow of $A\left(\frac{\partial}{\partial x^{1}}\right)$ is invariant with respect to the fiber homotheties of $J^{r}\left(\mathbb{R}^{m, n}\right)$ because $\frac{\partial}{\partial x^{1}}$ is invariant with respect to the fiber homotheties of $\mathbb{R}^{m, n}$.) Next we use the same arguments as in the proof of Lemma 1. For $J^{r} E^{*},\left(J^{r} E\right)^{*}$ and $\left(J^{r} E^{*}\right)^{*}$ in place of $J^{r} E$ we use the same method.

There is a natural involution (dualization) ()* $\mathcal{V H B}_{m, n} \rightarrow \mathcal{V} \mathcal{B}_{m, n}$, $E \mapsto E^{*}, f \mapsto\left(f^{-1}\right)^{*}$. So, using Proposition 2 and Theorem 1 we easily obtain the following versions of Theorem 1 .

TheOREM 2. For $m \geq 2, n \geq 1$ and $r \geq 0$ any $\mathcal{V} \mathcal{B}_{m, n}$-natural operator lifting a linear vector field $X$ on $E$ to a vector field $A(X)$ on $J^{r} E^{*}$ is a linear combination of $\mathcal{J}^{r} X^{*}$ and the Liouville vector field $L$ on $J^{r} E^{*}$, where $X^{*}$ is the linear vector field on $E^{*}$ dual to $X$ (if $f_{t}$ is the flow of $X$, then $\left(f_{t}^{-1}\right)^{*}$ is the flow of $\left.X^{*}\right)$.

TheOREM 3. For $m \geq 2, n \geq 1$ and $r \geq 0$ any $\mathcal{V} \mathcal{B}_{m, n}$-natural operator lifting a linear vector field $X$ on $E$ to a vector field $A(X)$ on $\left(J^{r} E\right)^{*}$ is a linear combination of $\left(\mathcal{J}^{r} X\right)^{*}$ and the Liouville vector field on $\left(J^{r} E\right)^{*}$.

TheOREM 4. For $m \geq 2, n \geq 1$ and $r \geq 0$ any $\mathcal{V} \mathcal{B}_{m, n}$-natural operator lifting a linear vector field $X$ on $E$ to a vector field $A(X)$ on $\left(J^{r} E^{*}\right)^{*}$ is a linear combination of $\left(\mathcal{J}^{r} X^{*}\right)^{*}$ and the Liouville vector field $L$ on $\left(J^{r} E^{*}\right)^{*}$.

\section{NATURAL OPERATORS LIFTING LINEAR VECTOR FIELDS FROM A VECTOR BUNDLE TO ITS VERTICAL $r$-JET PROLONGATION}

12. The vertical $r$-jet prolongation functor. Given a $\mathcal{V} \mathcal{B}_{m}$-object $p: E \rightarrow M$ the vertical $r$-jet prolongation $J_{\mathrm{v}}^{r} E$ of $E$ is the vector bundle

$$
J_{\mathrm{v}}^{r} E=\left\{j_{x}^{r} \sigma \mid \sigma: M \rightarrow E_{x}, x \in M\right\}
$$

over $M$. Every $\mathcal{V} \mathcal{B}_{m}$-map $f: E_{1} \rightarrow E_{2}$ covering $f: M_{1} \rightarrow M_{2}$ induces a vector bundle map $J_{\mathrm{v}}^{r} f: J_{\mathrm{v}}^{r} E_{1} \rightarrow J_{\mathrm{v}}^{r} E_{2}$ covering $\underline{f}$ such that

$$
J_{\mathrm{v}}^{r} f\left(j_{x}^{r} \sigma\right)=j_{\underline{f}(x)}^{r}\left(f \circ \sigma \circ \underline{f}^{-1}\right), \quad j_{x}^{r} \sigma \in J_{\mathrm{v}}^{r} E_{1} .
$$

The functor $J_{\mathrm{v}}^{r}: \mathcal{V B}_{m} \rightarrow \mathcal{V} \mathcal{B}_{m}$ is a fiber product preserving vector gauge bundle functor. 
We shall identify $\left(J_{\mathrm{v}}^{r}\right)_{0}\left(\mathbb{R}^{m, n}\right)$ and $\times^{n} J_{0}^{r}\left(\mathbb{R}^{m}, \mathbb{R}\right)$ via $j_{0}^{r}(\sigma) \mapsto\left(j_{0}^{r} \sigma^{l}\right)_{l=1}^{n}$, $\sigma=\left(\sigma^{1}, \ldots, \sigma^{n}\right): \mathbb{R}^{m} \rightarrow \mathbb{R}^{n}=\left(\mathbb{R}^{m, n}\right)_{0}$.

13. Examples of natural operators $T_{\operatorname{lin} \mid \mathcal{V B}} \mathcal{B}_{m, n} \rightsquigarrow T J_{\mathrm{v}}^{r}$. A natural operator $A: T_{\operatorname{lin} \mid \mathcal{V} \mathcal{B}_{m, n}} \rightsquigarrow T J_{\mathrm{v}}^{r}$ is a $\mathcal{V} \mathcal{B}_{m, n}$-invariant family of regular operators $A: \mathcal{X}_{\operatorname{lin}}(E) \rightarrow \mathcal{X}\left(J_{\mathrm{v}}^{r} E\right)$ for any $\mathcal{V} \mathcal{B}_{m, n}$-object $E$.

EXAmple 3 (The flow operator). Let $X$ be a linear vector field on a $\mathcal{V} \mathcal{B}_{m, n}$-object $E$. The vector field $\mathcal{J}_{\mathrm{v}}^{r} X$ on $J_{\mathrm{v}}^{r} E$ corresponding to the flow $J_{\mathrm{v}}^{r}\left(\mathrm{Fl}_{t}^{X}\right)$ is called the flow prolongation of $X$. The correspondence $\mathcal{J}_{\mathrm{v}}^{r}$ : $T_{\operatorname{lin} \mid \mathcal{V} \mathcal{B}_{m, n}} \rightsquigarrow T J_{\mathrm{v}}^{r}, X \mapsto \mathcal{J}_{\mathrm{v}}^{r} X$, is a natural operator.

EXAMPLE 4 (The Liouville vector field). Let $E$ be a $\mathcal{V} \mathcal{B}_{m, n}$-object. Let $L$ be the Liouville vector field on the vector bundle $J_{\mathrm{v}}^{r} E$. The correspondence $L: T_{\text {lin } \mid \mathcal{V} \mathcal{B}_{m, n}} \rightsquigarrow T J_{\mathrm{v}}^{r}, X \mapsto L$, is a natural operator.

ExAmple 5. Let $s=0, \ldots, r$. Given a linear vector field $X$ on a $\mathcal{V} \mathcal{B}_{m, n^{-}}$ object $E$ covering a vector field $\underline{X}$ on $M$ we define a vertical vector field $V^{\langle s\rangle}(X)$ on $J_{\mathrm{v}}^{r} E$ as follows. Let $y=j_{x}^{r} \sigma \in J_{\mathrm{v}}^{r} E, \sigma: M \rightarrow E_{x}, x \in M$. We put

$$
V^{\langle s\rangle}(X)(y)=\left(y, j_{x}^{r}\left(\underline{X}^{(s)} \sigma(x)\right)\right) \in\{y\} \times\left(J_{\mathrm{v}}^{r}\right)_{x} E=V_{y} J_{\mathrm{v}}^{r} E \subset T_{y} J_{\mathrm{v}}^{r} E,
$$

where $\underline{X}^{(s)}=X \circ \ldots \circ X$ (s times) and $\underline{X}^{(s)} \sigma(x): M \rightarrow E_{x}$ is the constant map. We see that $V^{\langle s\rangle}(X)$ are linear vector fields on the vector bundle $J_{\mathrm{v}}^{r} E$. The correspondence $V^{\langle s\rangle}: T_{\operatorname{lin} \mid \mathcal{V} \mathcal{B}_{m, n}} \rightsquigarrow T J_{\mathrm{v}}^{r}$ is a natural operator.

14. The main result of the second part. The main result in the second part is the following classification theorem.

Theorem 5. Let $m \geq 2, n \geq 1$ and $r \geq 1$ be integers. Any natural operator $A: T_{\operatorname{lin} \mid \mathcal{V} \mathcal{B}_{m, n}} \rightsquigarrow T J_{\mathrm{v}}^{r}$ is a linear combination with uniquely determined real coefficients of the flow operator $\mathcal{J}_{\mathrm{v}}^{r}$, the Liouville vector field $L$ and the natural operators $V^{\langle s\rangle}$ for $s=0, \ldots, r$.

The proof is a modification of the one of Theorem 1. It will occupy Sections 15-21.

Corollary 2. Let $m \geq 2, n \geq 1$ and $r \geq 1$ be integers. Any natural linear operator $A: T_{\operatorname{lin} \mid \mathcal{V} \mathcal{B}_{m, n}} \rightsquigarrow T J_{\mathrm{v}}^{r}$ is a linear combination with uniquely determined real coefficients of the flow operator $\mathcal{J}_{\mathrm{v}}^{r}$ and $V^{\langle 1\rangle}$.

\section{A reducibility lemma}

Lemma 5. Every natural operator $A: T_{\operatorname{lin} \mid \mathcal{V} \mathcal{B}_{m, n}} \rightsquigarrow T J_{\mathrm{v}}^{r}$ is uniquely determined by the restriction $\widetilde{A}=A\left(\frac{\partial}{\partial x^{1}}\right) \mid\left(J_{\mathrm{v}}^{r}\right)_{0}\left(\mathbb{R}^{m, n}\right)$ of $A\left(\frac{\partial}{\partial x^{1}}\right)$ to the fiber $\left(J_{\mathrm{v}}^{r}\right)_{0}\left(\mathbb{R}^{m, n}\right)$ of $J_{\mathrm{v}}^{r}\left(\mathbb{R}^{m, n}\right)$ over $0 \in \mathbb{R}^{m}$.

Proof. In the proof of Lemma 1 we replace $J^{r}$ by $J_{\mathrm{v}}^{r}$. 


\section{A decomposition lemma}

Lemma 6. Let $A: T_{\operatorname{lin} \mid \mathcal{V} \mathcal{B}_{m, n}} \rightsquigarrow T J_{\mathrm{v}}^{r}$ be a natural operator. Then there exists $\alpha \in \mathbb{R}$ such that $A-\alpha \mathcal{J}_{\mathrm{v}}^{r}$ is a vertical type operator.

Proof. In the proof of Lemma 2 we replace $J^{r}$ by $J_{\mathrm{v}}^{r}$.

17. Natural operators $T_{\operatorname{lin} \mid \mathcal{V} \mathcal{B}_{m, n}} \rightsquigarrow T J_{\mathrm{v}}^{r}$ of vertical type. By Lemma 6 , Theorem 5 is a consequence of the following fact.

Proposition 3. Let $m \geq 2, n \geq 1$ and $r \geq 1$ be integers. Let $A$ : $T_{\text {lin } \mid \mathcal{V} \mathcal{B}_{m, n}} \rightsquigarrow T J_{\mathrm{v}}^{r}$ be a natural operator of vertical type. There exist uniquely determined numbers $\gamma, \alpha_{0}, \ldots, \alpha_{r} \in \mathbb{R}$ such that $A=\gamma L+\sum_{s=0}^{r} \alpha_{s} V^{\langle s\rangle}$.

The proof will occupy Sections 18-21.

\section{Some preparation}

Lemma 7. Let $A: T_{\text {lin } \mid \mathcal{V} \mathcal{B}_{m, n}} \rightsquigarrow T J_{\mathrm{v}}^{r}$ be a natural operator of vertical type. Define $\bar{A}:\left(J_{\mathrm{v}}^{r}\right)_{0}\left(\mathbb{R}^{m, n}\right) \rightarrow\left(J_{\mathrm{v}}^{r}\right)_{0}\left(\mathbb{R}^{m, n}\right)$ by

$$
\widetilde{A}(y)=(y, \bar{A}(y)) \in\left(J_{\mathrm{v}}^{r}\right)_{0}\left(\mathbb{R}^{m, n}\right) \times\left(J_{\mathrm{v}}^{r}\right)_{0}\left(\mathbb{R}^{m, n}\right) \cong\left(V J_{\mathrm{v}}^{r}\right)_{0}\left(\mathbb{R}^{m, n}\right),
$$

$y \in\left(J_{\mathrm{v}}^{r}\right)_{0}\left(\mathbb{R}^{m, n}\right)$, where $\widetilde{A}$ is as in Lemma 5 . Then $A$ is uniquely determined by $\bar{A}$. Moreover, $\bar{A}$ is linear and satisfies the following conditions:

(i) For any $\mathcal{V} \mathcal{B}_{m, n}-\operatorname{map} f: \mathbb{R}^{m, n} \rightarrow \mathbb{R}^{m, n}$ preserving $\operatorname{germ}_{0}\left(\frac{\partial}{\partial x^{1}}\right)$,

$$
\left(J_{\mathrm{v}}^{r}\right)_{0} f \circ \bar{A}=\bar{A} \circ\left(J_{\mathrm{v}}^{r}\right)_{0} f .
$$

(ii) For any $\beta \in(\mathbb{N} \cup\{0\})^{m}$ with $|\beta| \leq r$ and any $l=1, \ldots, n$,

$$
\bar{A}\left(0, \ldots, 0, j_{0}^{r} x^{\beta}, 0, \ldots, 0\right)=\left(\sum_{|\sigma| \leq|\beta|} c_{\sigma}^{\beta, l, k} j_{0}^{r} x^{\sigma}\right)_{k=1}^{n}
$$

for some $c_{\sigma}^{\beta, l, k} \in \mathbb{R}$, where the sums are over all $\sigma \in(\mathbb{N} \cup\{0\})^{m}$ with $|\sigma| \leq|\beta|,\left(0, \ldots, 0, j_{0}^{r} x^{\beta}, 0, \ldots, 0\right) \in\left(J_{\mathrm{v}}^{r}\right)_{0}\left(\mathbb{R}^{m, n}\right), j_{0}^{r} x^{\beta}$ in position l.

Proof. In the proof of Lemma 3 we replace $J^{r}$ by $J_{\mathrm{v}}^{r}$.

19. The main lemma. By Lemma $7, A$ is uniquely determined by $\bar{A}$. So, Proposition 3 is a consequence of the following lemma.

Lemma 8. Let $r \geq 0, m \geq 2$ and $n \geq 1$ be integers. Let $\bar{A}:\left(J_{\mathrm{v}}^{r}\right)_{0}\left(\mathbb{R}^{m, n}\right)$ $\rightarrow\left(J_{\mathrm{v}}^{r}\right)_{0}\left(\mathbb{R}^{m, n}\right)$ be a linear map satisfying conditions (i) and (ii) of Lemma 7 . Then there are (uniquely determined for $r \geq 1$ ) numbers $\gamma, \alpha_{0}, \ldots, \alpha_{r}$ $\in \mathbb{R}$ such that

$$
\bar{A}=\gamma \operatorname{id}_{\left(J_{v}^{r}\right)_{0}\left(\mathbb{R}^{m, n}\right)}+\sum_{s=0}^{r} \alpha_{s} W^{\langle s\rangle},
$$


where $W^{\langle s\rangle}:\left(J_{\mathrm{v}}^{r}\right)_{0}\left(\mathbb{R}^{m, n}\right) \rightarrow\left(J_{\mathrm{v}}^{r}\right)_{0}\left(\mathbb{R}^{m, n}\right), W^{\langle s\rangle}\left(j_{0}^{r}(\sigma)\right)=j_{0}^{r}\left(\frac{\partial^{s}}{\partial\left(x^{1}\right)^{s}} \sigma(0)\right)$, $\sigma: \mathbb{R}^{m} \rightarrow \mathbb{R}^{n}=\left(\mathbb{R}^{m, n}\right)_{0}$.

If $r \geq 1$ then $\operatorname{id}_{\left(J_{\mathrm{v}}^{r}\right)_{0}\left(\mathbb{R}^{m, n}\right)}$ and $W^{\langle s\rangle}$ for $s=0, \ldots, r$ are linearly independent. So, it is sufficient to prove the existence of $\gamma, \alpha_{0}, \ldots, \alpha_{r}$ such that $\bar{A}=\gamma \operatorname{id}_{\left(J_{v}^{r}\right)_{0}\left(\mathbb{R}^{m, n}\right)}+\sum_{s=0}^{r} \alpha_{s} W^{\langle s\rangle}$.

We will prove the existence of $\gamma, \alpha_{0}, \ldots, \alpha_{r}$ by induction on $r$.

20. The case $r=0$. In Section 9 we replace $J^{r}$ by $J_{\mathrm{v}}^{r}$.

21. The inductive step. Assume that Lemma 8 is true for $r=r_{0}$. Let $r=r_{0}+1$.

We modify Steps $1-5$ of Section 10 with $J_{\mathrm{v}}^{r}$ playing the role of $J^{r}$. The most important change is in Step 1. We do not prove (2). By replacing $\bar{A}$ by $\bar{A}-c_{(0, \ldots, 0)}^{1} W^{\langle r\rangle}$ without loss of generality we can assume (2). Then it is sufficient to prove that $B=\gamma \operatorname{id}_{\left(J_{\mathrm{v}}^{r}\right)_{0}\left(\mathbb{R}^{m, n}\right)}+\sum_{s=0}^{r-1} \alpha_{s} W^{\langle s\rangle}$ for some $\gamma, \alpha_{0}, \ldots, \alpha_{r-1} \in \mathbb{R}$. Moreover, in Step 3 in the case $r=1$ we can assume that $\gamma=c$, where $c$ is as in (2) with $J_{\mathrm{v}}^{r}$ playing the role of $J^{r}$.

\section{Some versions of Theorem 5}

Proposition 4. Let $A$ be a $\mathcal{V B}_{m, n}$-natural operator lifting a linear vector field $X$ on a vector bundle $E$ to a vector field $A(X)$ on $J_{\mathrm{v}}^{r} E\left(\right.$ or $J_{\mathrm{v}}^{r} E^{*}$ or $\left(J_{\mathrm{v}}^{r} E\right)^{*}$ or $\left.\left(J_{\mathrm{v}}^{r} E^{*}\right)^{*}\right)$. Then $A(X)$ is always a linear vector field.

Proof. In the proof of Proposition 2 we replace $J^{r}$ by $J_{\mathrm{v}}^{r}$.

Using Proposition 3 and Theorem 5 we easily obtain the following versions of Theorem 5 .

Theorem 6. For $m \geq 2, n \geq 1$ and $r \geq 1$ any $\mathcal{V} \mathcal{B}_{m, n}$-natural operator lifting a linear vector field $X$ on $E$ to a vector field on $J_{\mathrm{v}}^{r} E^{*}$ is a linear combination of $\mathcal{J}_{\mathrm{v}}^{r} X^{*}$, the Liouville vector field $L$ on $J_{\mathrm{v}}^{r} E^{*}$ and $V^{\langle s\rangle}\left(X^{*}\right)$ for $s=0, \ldots, r$.

Theorem 7. For $m \geq 2, n \geq 1$ and $r \geq 1$ any $\mathcal{V B}_{m, n}$-natural operator lifting a linear vector field $X$ on $E$ to a vector field on $\left(J_{\mathrm{v}}^{r} E\right)^{*}$ is a linear combination of $\left(\mathcal{J}_{\mathrm{v}}^{r} X\right)^{*}$, the Liouville vector field $L$ on $\left(J_{\mathrm{v}}^{r} E\right)^{*}$ and $\left(V^{\langle s\rangle}(X)\right)^{*}$ for $s=0, \ldots, r$.

TheOREM 8. For $m \geq 2, n \geq 1$ and $r \geq 1$ any $\mathcal{V} \mathcal{B}_{m, n}$-natural operator lifting a linear vector field $X$ on $E$ to a vector field on $\left(J_{\mathrm{v}}^{r} E^{*}\right)^{*}$ is a linear combination of $\left(\mathcal{J}_{\mathrm{v}}^{r} X^{*}\right)^{*}$, the Liouville vector field $L$ on $\left(J_{\mathrm{v}}^{r} E^{*}\right)^{*}$ and $\left(V^{\langle s\rangle}\left(X^{*}\right)\right)^{*}$ for $s=0, \ldots, r$. 


\section{NATURAL LINEAR OPERATORS LIFTING LINEAR VECTOR FIELDS FROM A VECTOR BUNDLE TO ITS $[r]$-JET PROLONGATION}

23. The $[r]$-jet prolongation functor. Let $p: E \rightarrow M$ be a $\mathcal{V B}_{m^{-}}$ object. For any $x \in M$ we have a unital associative algebra homomorphism $t_{x}^{[r]}: J_{x}^{r}(M, \mathbb{R}) \rightarrow \operatorname{gl}\left(J_{x}^{r}(M, \mathbb{R})\right)$ given by

$$
t_{x}^{[r]}\left(j_{x}^{r} \gamma\right)\left(j_{x}^{r} \eta\right)=j_{x}^{r}(\gamma \eta)-j_{x}^{r}(\eta(x) \gamma)+j_{x}^{r}(\eta(x) \gamma(x)),
$$

$j_{x}^{r} \eta, j_{x}^{r} \gamma \in J_{x}^{r}(M, \mathbb{R}), \eta(x), \gamma(x): M \rightarrow \mathbb{R}$ are constant maps. We have a vector bundle

$$
J^{[r]} E=\bigcup_{x \in M} \operatorname{Hom}_{t_{x}^{[r]}}\left(J^{r} \mathcal{C}_{x}^{\infty, \mathrm{f} . \mathrm{l}}(E), J_{x}^{r}(M, \mathbb{R})\right)
$$

over $M$. Here $\operatorname{Hom}_{t_{x}^{[r]}}\left(J^{r} \mathcal{C}_{x}^{\infty, f . l}(E), J_{x}^{r}(M, \mathbb{R})\right)$ is the vector space of all module homomorphisms over $t_{x}^{[r]}: J_{x}^{r}(M, \mathbb{R}) \rightarrow \operatorname{gl}\left(J_{x}^{r}(M, \mathbb{R})\right)$ from the (free) $J_{x}^{r}(M, \mathbb{R})$-module $J^{r} \mathcal{C}_{x}^{\infty, f . l}(E)$ of $r$-jets at $x$ of germs at $x$ of fiber linear maps $E \rightarrow \mathbb{R}$ into the $\operatorname{gl}\left(J_{x}^{r}(M, \mathbb{R})\right)$-module $J_{x}^{r}(M, \mathbb{R})$. We call $J^{[r]} E$ the $[r]$ jet prolongation of $E$. Every $\mathcal{V} \mathcal{B}_{m}$-map $f: E_{1} \rightarrow E_{2}$ covering $\underline{f}: M_{1} \rightarrow M_{2}$ induces a vector bundle map $J^{[r]} f: J^{[r]} E_{1} \rightarrow J^{[r]} E_{2}$ covering $\underline{f}$ such that

$$
J^{[r]} f(\Phi)\left(j_{\underline{f}(x)}^{r} \xi\right)=J^{r}\left(\underline{f}, \mathrm{id}_{\mathbb{R}}\right) \circ \Phi\left(j_{x}^{r}(\xi \circ f)\right)
$$

for any $\Phi \in \operatorname{Hom}_{t_{x}^{[r]}}\left(J^{r} \mathcal{C}_{x}^{\infty, f . l}\left(E_{1}\right), J_{x}^{r}\left(M_{1}, \mathbb{R}\right)\right), x \in M_{1}$ and any fiber linear $\operatorname{map} \xi: E_{2} \rightarrow \mathbb{R}$. The correspondence $J^{[r]}: \mathcal{V} \mathcal{B}_{m} \rightarrow \mathcal{V} \mathcal{B}_{m}$ is a fiber product preserving gauge bundle functor of order $r$.

REmark 1. One can show that $J^{r} E$ and $J_{\mathrm{v}}^{r} E$ can be constructed similarly to $J^{[r]} E$ using some other algebra homomorphisms $t_{x}: J_{x}^{r}(M, \mathbb{R}) \rightarrow$ $\operatorname{gl}\left(J_{x}^{r}(M, \mathbb{R})\right)$ in place of $t_{x}^{[r]}$. This justifies the name of $[r]$-jet prolongation. If $r \geq 3$ and $m \geq 2$ then only $J^{r} E, J_{\mathrm{v}}^{r} E$ and $J^{[r]} E$ admit such a construction. This will be presented in my next paper, currently in preparation.

Similarly to the usual jet projections $J^{r} E \rightarrow J^{r-1} E$ or $J_{\mathrm{v}}^{r} E \rightarrow J_{\mathrm{v}}^{r-1} E$ we have the canonical projection $\pi_{[r-1]}^{[r]}: J^{[r]} E \rightarrow J^{[r-1]} E$ for $r \geq 1$ (and $\pi_{[s]}^{[r]}=\pi_{[r-1]}^{[r]} \circ \ldots \circ \pi_{[s]}^{[s+1]}$ for $\left.s=0, \ldots, r-1\right)$. Indeed, by standard verification, any module homomorphism $\Phi: J^{r} \mathcal{C}_{x}^{\infty, f . l}(E) \rightarrow J_{x}^{r}(M \mathbb{R})$ over $t_{x}^{[r]}: J_{x}^{r}(M, \mathbb{R}) \rightarrow \operatorname{gl}\left(J_{x}^{r}(M, \mathbb{R})\right)$ can be factorized by a linear map $\underline{\Phi}: J^{r-1} \mathcal{C}_{x}^{\infty}$,f.l $(E) \rightarrow J_{x}^{r-1}(M, \mathbb{R})$ which is a module homomorphism over $t_{x}^{[r-1]}: J_{x}^{r-1}(M, \mathbb{R}) \rightarrow \operatorname{gl}\left(J_{x}^{r-1}(M, \mathbb{R})\right)$. Then we put $\pi_{[r-1]}^{[r]}(\Phi)=\underline{\Phi}$ for any $\Phi$ as above. 
We shall identify the vector spaces $J_{0}^{[r]}\left(\mathbb{R}^{m, n}\right)$ and $\times^{n} J_{0}^{r}\left(\mathbb{R}^{m}, \mathbb{R}\right)$ via the linear isomorphism $\Phi \mapsto\left(\Phi\left(j_{0}^{r} y^{l}\right)\right)_{l=1}^{n}$, where $y^{1}, \ldots, y^{n}$ are the usual fiber coordinates on $\mathbb{R}^{m, n}$ which are fiber linear maps $\mathbb{R}^{m, n} \rightarrow \mathbb{R}$.

24. Examples of natural linear operators $T_{\operatorname{lin} \mid \mathcal{V} \mathcal{B}_{m, n}} \rightsquigarrow T J^{[r]}$. A natural linear operator $A: T_{\operatorname{lin} \mid \mathcal{V} \mathcal{B}_{m, n}} \rightsquigarrow T J^{[r]}$ is an $\mathcal{V} \mathcal{B}_{m, n}$-invariant family of $\mathbb{R}$-linear operators $A: \mathcal{X}_{\text {lin }}(E) \rightarrow \mathcal{X}\left(J^{[r]} E\right)$ for any $\mathcal{V} \mathcal{B}_{m, n}$-object $E$.

EXAmple 6 (The flow operator). Let $X$ be a linear vector field on a $\mathcal{V} \mathcal{B}_{m, n}$-object $E$. The vector field $\mathcal{J}^{[r]} X$ on $J^{[r]} E$ corresponding to the flow $J^{[r]}\left(\mathrm{Fl}_{t}^{X}\right)$ is called the flow prolongation of $X$. The correspondence $\mathcal{J}^{[r]}$ : $T_{\operatorname{lin} \mid \mathcal{V} \mathcal{B}_{m, n}} \rightsquigarrow T J^{[r]}, X \mapsto \mathcal{J}^{[r]} X$, is a natural linear operator.

EXAMPLE 7. Let $s=0, \ldots, r$. Given a linear vector field $X$ on a $\mathcal{V} \mathcal{B}_{m, n^{-}}$ object $E$ covering a vector field $\underline{X}$ on $M$ and a module homomorphism $\Phi: J^{r} \mathcal{C}_{x}^{\infty, f . l}(E) \rightarrow J_{x}^{r}(M, \mathbb{R})$ over $t_{x}^{[r]}: J_{x}^{r}(M, \mathbb{R}) \rightarrow \operatorname{gl}\left(J_{x}^{r}(M, \mathbb{R})\right)$ (i.e. $\Phi \in$ $\left.J_{x}^{[r]} E, x \in M\right)$ we have a linear map $\Phi_{X}^{(s)}: J^{r} \mathcal{C}_{x}^{\infty, f . l}(E) \rightarrow J_{x}^{r}(M, \mathbb{R})$ given by

$$
\Phi_{X}^{(s)}(\sigma)=j_{x}^{r}\left(\underline{X}^{(s)} \gamma(x)\right)
$$

$\sigma \in J^{r} \mathcal{C}_{x}^{\infty, f . l}(E), \gamma: M \rightarrow \mathbb{R}, j_{x}^{r} \gamma=\Phi(\sigma), X^{(s)}=X \circ \ldots \circ X$ ( $s$ times), $\underline{X}^{(s)} \gamma(x): M \rightarrow \mathbb{R}$ being the constant map. Unfortunately, the linear maps $\Phi_{X}^{(s)}: J^{r} \mathcal{C}_{x}^{\infty, f . l}(E) \rightarrow J_{x}^{r}(M, \mathbb{R})$ are module homomorphisms over $t_{x}^{[r]}: J_{x}^{r}(M, \mathbb{R}) \rightarrow \operatorname{gl}\left(J_{x}^{r}(M, \mathbb{R})\right)$ only for $s=0$ and $s=1$, as is easily verified. For $s=2, \ldots, r$ the map $\Phi_{X}^{(s)}: J^{r} \mathcal{C}_{x}^{\infty, f . l}(E) \rightarrow J_{x}^{r}(M, \mathbb{R})$ is a module homomorphism over $t_{x}^{[r]}: J_{x}^{r}(M, \mathbb{R}) \rightarrow \operatorname{gl}\left(J_{x}^{r}(M, \mathbb{R})\right)$ if $\Phi \in \operatorname{ker}\left(\pi_{[s-1]}^{[r]}\right) \subset J^{[r]} E$. Consequently, only for $s=0$ and $s=1$ do we have vertical vector fields $U^{(s)}(X)$ on $J^{[r]} E$, given by

$$
U^{(s)}(X)_{\Phi}=\left(\Phi, \Phi_{X}^{(s)}\right) \in\{\Phi\} \times J_{x}^{[r]} E=V_{\Phi} J^{[r]} E,
$$

$\Phi \in J_{x}^{[r]} E, x \in M$. For $s=2, \ldots, r$ we have $U^{(s)}(X)$ only on $\operatorname{ker}\left(\pi_{[s-1]}^{[r]}\right) \subset$ $J^{[r]} E$. The correspondence $U=U^{(1)}: T_{\operatorname{lin} \mid \mathcal{V} \mathcal{B}_{m, n}} \rightsquigarrow T J^{[r]}$ as above is a natural linear operator.

25. The main result of the third part. The main result of the third part is the following classification theorem.

Theorem 9. Let $m \geq 2, n \geq 1$ and $r \geq 1$ be integers. Any natural linear operator $A: T_{\operatorname{lin} \mid \mathcal{V} \mathcal{B}_{m, n}} \rightsquigarrow T J^{[r]}$ is a linear combination with uniquely determined real coefficients of the flow operator $\mathcal{J}^{[r]}$ and the operator $U$.

The proof is a modification of the one of Theorem 1. It will occupy Sections 26-32. 


\section{A reducibility lemma}

Lemma 9. Let $A: T_{\operatorname{lin} \mid \mathcal{V} \mathcal{B}_{m, n}} \rightsquigarrow T J^{[r]}$ be a natural (not necessarily linear) operator. The operator $A$ is uniquely determined by the restriction $\widetilde{A}=A\left(\frac{\partial}{\partial x^{1}}\right) \mid J_{0}^{[r]}\left(\mathbb{R}^{m, n}\right)$ of $A\left(\frac{\partial}{\partial x^{1}}\right)$ to the fiber $J_{0}^{[r]}\left(\mathbb{R}^{m, n}\right)$ of $J^{[r]}\left(\mathbb{R}^{m, n}\right)$ over $0 \in \mathbb{R}^{m}$.

Proof. In the proof of Lemma 1 we replace $J^{r}$ by $J^{[r]}$.

\section{A decomposition lemma}

Lemma 10. Let $A: T_{\operatorname{lin} \mid \mathcal{V} \mathcal{B}_{m, n}} \rightsquigarrow T J^{[r]}$ be a natural (not necessarily linear) operator. Then there exists $\alpha \in \mathbb{R}$ such that $A-\alpha \mathcal{J}^{[r]}$ is a vertical type operator.

Proof. In the proof of Lemma 2 we replace $J^{r}$ by $J^{[r]}$.

28. The natural linear operators $T_{\operatorname{lin} \mid \mathcal{V} \mathcal{B}_{m, n}} \rightsquigarrow T J^{[r]}$ of vertical type. By Lemma 10, Theorem 9 is a consequence of the following fact.

Proposition 5. Let $m \geq 2, n \geq 1$ and $r \geq 1$ be integers. Let $A$ : $T_{\text {lin } \mid \mathcal{V} \mathcal{B}_{m, n}} \rightsquigarrow T J^{[r]}$ be a natural linear operator of vertical type. Then there exists a number $\gamma \in \mathbb{R}$ such that $A=\gamma U$.

The proof will occupy Sections 29-32.

\section{Some preparation}

Lemma 11. Let $A: T_{\operatorname{lin} \mid \mathcal{V} \mathcal{B}_{m, n}} \rightsquigarrow T J^{[r]}$ be a natural linear operator of vertical type. Define $\bar{A}: J_{0}^{[r]}\left(\mathbb{R}^{m, n}\right) \rightarrow J_{0}^{[r]}\left(\mathbb{R}^{m, n}\right)$ by

$$
\widetilde{A}(y)=(y, \bar{A}(y)) \in J_{0}^{[r]}\left(\mathbb{R}^{m, n}\right) \times J_{0}^{[r]}\left(\mathbb{R}^{m, n}\right) \cong\left(V J^{[r]}\right)_{0}\left(\mathbb{R}^{m, n}\right),
$$

$y \in J_{0}^{[r]}\left(\mathbb{R}^{m, n}\right)$, where $\widetilde{A}$ is as in Lemma 9 . Then $A$ is uniquely determined by $\bar{A}$. Moreover, $\bar{A}$ is linear and satisfies the following conditions:

(i) For any $\mathcal{V} \mathcal{B}_{m, n}-$ map $f: \mathbb{R}^{m, n} \rightarrow \mathbb{R}^{m, n}$ preserving $\operatorname{germ}_{0}\left(\frac{\partial}{\partial x^{1}}\right)$,

$$
J_{0}^{[r]} f \circ \bar{A}=\bar{A} \circ J_{0}^{[r]} f .
$$

(ii) For any $\beta \in(\mathbb{N} \cup\{0\})^{m}$ with $|\beta| \leq r$ and any $l=1, \ldots, n$,

$$
\bar{A}\left(0, \ldots, 0, j_{0}^{r} x^{\beta}, 0, \ldots, 0\right)=\left(\sum_{|\sigma|=|\beta|-1} c_{\sigma}^{\beta, l, k} j_{0}^{r} x^{\sigma}\right)_{k=1}^{n}
$$

for some $c_{\sigma}^{\beta, l, k} \in \mathbb{R}$, where the sums are over all $\sigma \in(\mathbb{N} \cup\{0\})^{m}$ with $|\sigma|=|\beta|-1,\left(0, \ldots, 0, j_{0}^{r} x^{\beta}, 0, \ldots, 0\right) \in J_{0}^{[r]}\left(\mathbb{R}^{m, n}\right), j_{0}^{r} x^{\beta}$ in position $l$.

Proof. In the proof of Lemma 3 we replace $J^{r}$ by $J^{[r]}$. In the proof of (ii) we use additionally the fact that $A$ is a linear operator. 
30. The main lemma. By Lemma $11, A$ is uniquely determined by $\bar{A}$. So, Proposition 5 is a consequence of the following lemma.

LEMma 12. Let $m \geq 2, n \geq 1$ and $r \geq 0$ be integers. Let $\bar{A}: J_{0}^{[r]}\left(\mathbb{R}^{m, n}\right)$ $\rightarrow J_{0}^{[r]}\left(\mathbb{R}^{m, n}\right)$ be a linear map satisfying conditions (i) and (ii) of Lemma 11 . Then there is $\gamma \in \mathbb{R}$ such that $\bar{A}=\gamma W$, where $W: J_{0}^{[r]}\left(\mathbb{R}^{m, n}\right) \rightarrow J_{0}^{[r]}\left(\mathbb{R}^{m, n}\right)$, $W\left(j_{0}^{r}(\sigma)\right)=j_{0}^{r}\left(\frac{\partial}{\partial x^{1}} \sigma(0)\right), \sigma: \mathbb{R}^{m} \rightarrow \mathbb{R}^{n}=\left(\mathbb{R}^{m, n}\right)_{0}($ if $r=0$, then $\bar{A}=0)$.

We will prove the existence of $\gamma$ by induction on $r$.

31. The case $r=0$. This is a consequence of condition (ii).

32. The inductive step. Assume that Lemma 12 for $r=r_{0}$ is true. Let $r=r_{0}+1$.

We modify Steps $1-5$ of Section 10 with $J^{[r]}$ playing the role of $J^{r}$. The most important change is in Step 1. If $r \geq 2$ we have (2) because of condition (ii). If $r=1$ without loss of generality we can assume (2) upon replacing $\bar{A}$ by $\bar{A}-c_{(0, \ldots, 0)}^{1} W$. Additionally, by condition (ii) we have $c=0$ in (3) for $J^{[r]}$ in place of $J^{r}$.

Remark 2. We have not been able to prove Theorem 9 for $A$ not necessarily linear because we cannot obtain (2) with $J^{[r]}$ playing the role of $J^{r}$. This is because we do not know whether $U^{(s)}(X)$ for $s=2, \ldots, r$ are canonically extendable to the whole $J^{[r]} E$ (see Example 7 ). If they were then we could prove (similarly to Theorem 1 ) that any $A(X)$ is a linear combination of $\mathcal{J}^{[r]} X$, the Liuoville vector field $L$ and extended $U^{(s)}(X)$ for $s=0, \ldots, r$. If $U^{(s)}(X)$ are not extendable then it will probably be difficult to classify all natural operators $A(X)$ on $J^{[r]} E$.

\section{Some versions of Theorem 9}

Proposition 6. Let $A$ be a $\mathcal{V B}_{m, n}$-natural (not necessarily linear) operator lifting a linear vector field $X$ on a vector bundle $E$ to a vector field $A(X)$ on $J^{[r]} E\left(\right.$ or $J^{[r]} E^{*}$ or $\left(J^{[r]} E\right)^{*}$ or $\left.\left(J^{[r]} E^{*}\right)^{*}\right)$. Then $A(X)$ is always a linear vector field.

Proof. In the proof of Proposition 2 we replace $J^{r}$ by $J^{[r]}$.

Using Proposition 6 and Theorem 9 we easily obtain the following versions of Theorem 9.

TheOREM 10. For $m \geq 2, n \geq 1$ and $r \geq 1$ any $\mathcal{V B}_{m, n}$-natural linear operator lifting a linear vector field $X$ on $E$ to a vector field on $J^{[r]} E^{*}$ is a linear combination of $\mathcal{J}^{[r]} X^{*}$ and $U\left(X^{*}\right)$. 
TheOREM 11. For $m \geq 2, n \geq 1$ and $r \geq 1$ any $\mathcal{V} \mathcal{B}_{m, n}$-natural linear operator lifting a linear vector field $X$ on $E$ to a vector field on $\left(J^{[r]} E\right)^{*}$ is a linear combination of $\left(\mathcal{J}^{[r]} X\right)^{*}$ and $(U(X))^{*}$.

THEOREM 12. For $m \geq 2, n \geq 1$ and $r \geq 1$ any $\mathcal{V B}_{m, n}$-natural linear operator lifting a linear vector field $X$ on $E$ into a vector field on $\left(J^{[r]} E^{*}\right)^{*}$ is a linear combination of $\left(\mathcal{J}^{[r]} X^{*}\right)^{*}$ and $\left(U\left(X^{*}\right)\right)^{*}$.

\section{References}

[1] M. Doupovec, Natural operators transforming vector fields to the second order tangent bundle, Časopis Pěst. Mat. 115 (1990), 64-72.

[2] J. Gancarzewicz, Liftings of functions and vector fields to natural bundles, Dissertationes Math. 212 (1983).

[3] I. Kolář, P. W. Michor and J. Slovák, Natural Operations in Differential Geometry, Springer, Berlin, 1993.

[4] J. Kurek, On natural operators transforming vector fields on manifold to second iterated tangent bundle, Demonstratio Math. 25 (1992), 765-776.

[5] W. M. Mikulski, The jet prolongations of fibered fibered manifolds and the flow operator, Publ. Math. Debrecen 59 (2001), 441-458.

Institute of Mathematics

Jagiellonian University

Reymonta 4

30-059 Kraków, Poland

E-mail: mikulski@im.uj.edu.pl

Reçu par la Rédaction le 11.2.2003

Révisé le 2.4.2003 